\title{
Third-Order Nonlinear Susceptibilities of Dye Solutions Determined by Third-Harmonic Generation
}

\author{
W. Leupacher and A. Penzkofer \\ Naturwissenschaftliche Fakultät II - Physik, Universität, D-8400 Regensburg, \\ Fed. Rep. Germany
}

Received 8 August 1984/Accepted 28 August 1984

\begin{abstract}
The third-order nonlinear susceptibilities $\chi_{x x x x}^{(3)}\left(-\omega_{3} ; \omega_{1}, \omega_{1}, \omega_{1}\right)$ versus concentration are determined for rhodamine $6 \mathrm{G}$, fuchsin, and methylene blue in methanol. Third-harmonic generation of a modelocked Nd-glass laser is applied. The real and imaginary part of the nonlinear susceptibility are measured. A resonance enhancement due to absorption bands is observed.
\end{abstract}

PACS: $42.65,42.65 \mathrm{~B}$

Phasematched third-harmonic generation in dye solutions was studied in the past as potential process of efficient frequency tripling [1-6]. The conversion efficiency $\eta$ could be increased about a factor of 50 (fuchsin [1-4]) to 4000 (hexamethylindocarbocyanine iodide [5]) compared to the neat solvent. Phasematching was achieved by utilizing the anomalous dispersion of the refractive index of the dye solutions. The absolute conversion efficiency remained weak $(\eta \approx 2$ $\times 10^{-11}$ in fuchsin [2], $\eta \approx 2 \times 10^{-9}$ in hexamethylindocarbocyanine iodide [5] at $10^{7} \mathrm{~W} / \mathrm{cm}^{2}$ laser intensity) because of light absorption at the thirdharmonic frequency limiting the interaction length. The nonlinear hyperpolarizabilities of dye molecules were estimated and related to conjugated bonds and two-photon-resonances [5].

Here we determine the dependence of third-order nonlinear susceptibilities $\chi_{x x x x}^{(3)}\left(-\omega_{3} ; \omega_{1}, \omega_{1}, \omega_{1}\right)$ on dye concentration for rhodamine $6 \mathrm{G}$, fuchsin and methylene blue in methanol. The efficiency of conversion of picosecond light pulses of a mode-locked Ndglass laser to the third harmonic is measured. The nonlinear susceptibilities are deduced by comparison with calculations [7]. The absorption coefficients and refractive indices entering the calculations are separately measured $[8,9]$. The nonlinear susceptibilities comprise contributions from the solvent and the solute. The complex solute hyperpolarizabilities are determined and compared with the real solvent hyperpolarizability. The resonance enhancement is analyzed.

\section{Experiments}

The experiments are carried out with single picosecond light pulses from a passively mode-locked $\mathrm{Nd}$ phosphate glass laser. The experimental setup is identical to that of [7]. The most important characteristic of the arrangement is the location of the sample cell into a vacuum chamber and the use of cell windows with thickness equal to even integer multiples of the coherence length (achieved by tilting the sample to angle $\theta=6^{\circ}$ ). For this arrangement the detected thirdharmonic signal originates entirely from the liquid under investigation.

The theory of [7] is applied to calculate the nonlinear susceptibility values from energy conversion measurements. The measured energy conversion is given by the ratio of third-harmonic energy behind the end of the cell $W_{3}\left(d_{+}\right)$to the input fundamental energy in front of the entrance window $W_{1}\left(a_{-}\right)$

$\eta=\frac{W_{3}\left(d_{+}\right)}{W_{1}\left(a_{-}\right)}$. 
It is related to the input-pulse peak intensity $I_{01}\left(a_{-}\right)$ (Gaussian temporal and spatial pulse shape assumed), the nonlinear susceptibility $\chi_{x x x x}^{(3)}\left(-\omega_{3} ; \omega_{1}, \omega_{1}, \omega_{1}\right)$ (abreviated by $\chi^{(3)}$ ), the absorption coefficients $\alpha_{1}, \alpha_{3}$ and the refractive indices $n_{1}, n_{3}$ by

$$
\begin{aligned}
& \eta=\frac{\kappa}{3^{3 / 2}}\left|\chi^{(3)}\right|^{2} I_{10}^{2}\left(a_{-}\right) \\
& \frac{\left[1-R_{\perp 3}(c)\right]\left[1-R_{3 \perp}(d)\right]\left[1-R_{\perp 1}(a)\right]^{3}\left[1-R_{\perp 1}(b)\right]^{3}}{M^{2}(a) M^{2}(b)},
\end{aligned}
$$

$a, b, c, d$ denote the air-window, window-solution, solution-window, and window-air interfaces along the light path, respectively. The subscripts - and + indicate the positions in front of and behind the interface, respectively. $R_{\perp 1}$ and $R_{\perp 3}$ denote the light reflection at the interface. They are approximately given by $R_{\perp} \simeq R=\left(n_{-}-n_{+}\right)^{2} /\left(n_{-}+n_{+}\right)^{2} . M(a)$ and $M(b)$ describe the changes of the beam diameter of the input light at the air-window and the windowsolution interface, respectively. These magnification factors are $M=\cos \theta_{+} / \cos \theta_{-}$with $\sin \theta_{+}$ $=\left(n_{-} / n_{+}\right) \sin \theta_{-}$. The constant $\kappa$ is given by

$\kappa=\frac{4 \pi^{2} \tilde{v}_{3}^{2}\left\{\exp \left(-3 \alpha_{1} l_{B}\right)+\exp \left(-\alpha_{3} l_{B}\right)-2 \exp \left[-\left(\alpha_{3}+3 \alpha_{1}\right) l_{B} / 2\right] \cos \left(\Delta k l_{B}\right)\right\}}{n_{3} n_{1}^{3} \varepsilon_{0}^{2} c_{0}^{2}\left[\left(\alpha_{3}-3 \alpha_{1}\right)^{2} / 4+\Delta k^{2}\right]}$,

$\tilde{v}=\omega_{3} /\left(2 \pi c_{0}\right)=v_{3} / c_{0}=1 / \lambda_{3}$ is the wave number of the third-harmonic light. $c_{0}$ is the vacuum light velocity. $l_{B}$ is the inner cell length. The wave vector mismatch is $\Delta k=6 \pi \tilde{v}_{1}\left(n_{3}-n_{1}\right)\left(\tilde{v}_{1}=1 / \lambda_{1}, \lambda_{1}=1055 \mathrm{~nm}\right)$.

For transparent media $\left(\alpha_{1}=\alpha_{3}=0\right) \kappa$ reduces to

$\kappa=\frac{4 \pi^{2} \tilde{v}_{3}^{2}}{n_{3} n_{1}^{3} c_{0}^{2} \varepsilon_{0}^{2}} \frac{\sin ^{2}\left(\Delta k l_{B} / 2\right)}{(\Delta k / 2)^{2}}$

and the energy conversion $\eta$ is modulated with cell thickness $l_{B}$ (Maker fringes). The mean energy conversion (averaged over a modulation period) is defined as $\bar{\eta}=\left(\eta_{\max }+\eta_{\min }\right) / 2$. The neat solvent methanol has negligibly small absorption $\left(\alpha_{1}=0.112 \mathrm{~cm}^{-1}, \alpha_{3}=0\right)$ and the third-harmonic generation is governed by (4). For dye concentrations $C>10^{-2} \mathrm{~mol} / \mathrm{dm}^{3}$ the absorption coefficient $\alpha_{3}$ at the third-harmonic frequency is so large that the second and third terms in the curly brackets of (3) are negligible and $\kappa$ reduces to

$\kappa=\frac{4 \pi^{2} \tilde{v}_{3}^{2} \exp \left(-3 \alpha_{1} l_{B}\right)}{n_{3} n_{1}^{3} \varepsilon_{0}^{2} c_{0}^{2}\left[\left(\alpha_{3}-3 \alpha_{1}\right)^{2} / 4+\Delta k^{2}\right]}$.

The third-harmonic signal does not any longer exhibit an oscillatory length dependence. The effective interaction length is

$l_{\text {eff }}=\left[\left(\alpha_{3}-3 \alpha_{1}\right)^{2} / 4+\Delta k^{2}\right]^{-1 / 2}$.

Phasematched third-harmonic generation $(\Delta k=0$, $\left.n_{3}=n_{1}\right)$ is achievable in some dye solutions having

$\Delta\left[\bar{\eta} / I_{01}^{2}\left(a_{-}\right)\right] /\left[\bar{\eta} / I_{01}^{2}\left(a_{-}\right)\right] \approx \pm 0.3$ $\alpha_{3} \propto C$; S: solvent, D: dye). portional to the concentration. their $S_{0}-S_{1}$ absorption band between $\tilde{v}_{1}$ and $\tilde{v}_{3}$. But the effective interaction length remains generally short $\left[l_{\text {eff }}(\Delta k) \approx 2 / \alpha_{3}\right]$.

The absorption coefficients $\alpha_{1}$ and $\alpha_{3}$ of the dye solutions were measured with a spectro-photometer. Cell thicknesses down to $10 \mu \mathrm{m}$ were used. The refractive indices (relative to air) of the fused silica cell windows are $n_{1}=1.4497$ and $n_{3}=1.4765$ [10]. The refractive indices $n_{1}=1.3214$ and $n_{3}=1.3420$ $\left(T=25^{\circ} \mathrm{C}\right)$ of the solvent methanol were measured with a Pellin-Broca prism apparatus [8] while the refractive indices of the dye solutions were determined with a reflection technique [9]. The unknown susceptibility values $\left|\chi^{(3)}\right|$ are extracted from (2) by comparing the measured energy conversions with calculations.

\section{Results}

Methanolic solutions of rhodamine 6G, fuchsin (C.I. No.42510) and methylene blue (C.I. No. 52015) are investigated. The absorption spectra of the three dye

solutions are depicted in Fig. 1 (dye concentration $10^{-4} \mathrm{~mol} / \mathrm{dm}^{3}$ ). Rhodamine $6 \mathrm{G}$ has absorption peaks at $2 \omega_{1}$ and $\omega_{3}$, fuchsin absorbs at $2 \omega_{1}$ while methylene blue has no absorption maxima at $\omega_{1}, 2 \omega_{1}$ or $\omega_{3}$. The measured energy conversions versus dye concentration are depicted by the open circles in Figs. 2-4. The normalized quantity $\bar{\eta} / I_{01}^{2}\left(a_{-}\right)$is plotted. The uncertainty of data points is approximately

due to uncertainties in the energy conversion and intensity detection. At small dye concentrations up to some $10^{-3} \mathrm{~mol} / \mathrm{dm}^{3}$ the mean energy conversion reduces to approximately half the neat solvent value due to absorption of the generated third-harmonic light $\left[\exp \left(-\alpha_{3} l_{B}\right) \rightarrow 0\right.$ in (3)]. Then the energy conversion increases with concentration. In methylene blue a maximum is reached around $0.37 \mathrm{~mol} / \mathrm{dm}^{3}$. This maximum energy conversion coincides with phasematching $\left(\bar{\eta} \propto\left|\chi^{(3)}\right|^{2} /\left(\alpha_{3}^{2} / 4+\Delta \mathrm{k}^{2}\right),\left|\chi^{(3)}\right|=\left|\chi_{\mathrm{S}}^{(3)}+\chi_{\mathrm{D}}^{(3)}\right|,\left|\chi_{\mathrm{D}}^{(3)}\right| \propto C\right.$,

The absorption coefficients $\alpha_{1}$ and $\alpha_{3}$ versus concentration are displayed in Fig. 5. Within the experimental accuracy the absorption coefficients are pro-

The concentration dependence of the refractive indices is shown in Fig. 6. For rhodamine $6 \mathrm{G}$ and fuchsin the refractive indices grow linearly with concentration. In 


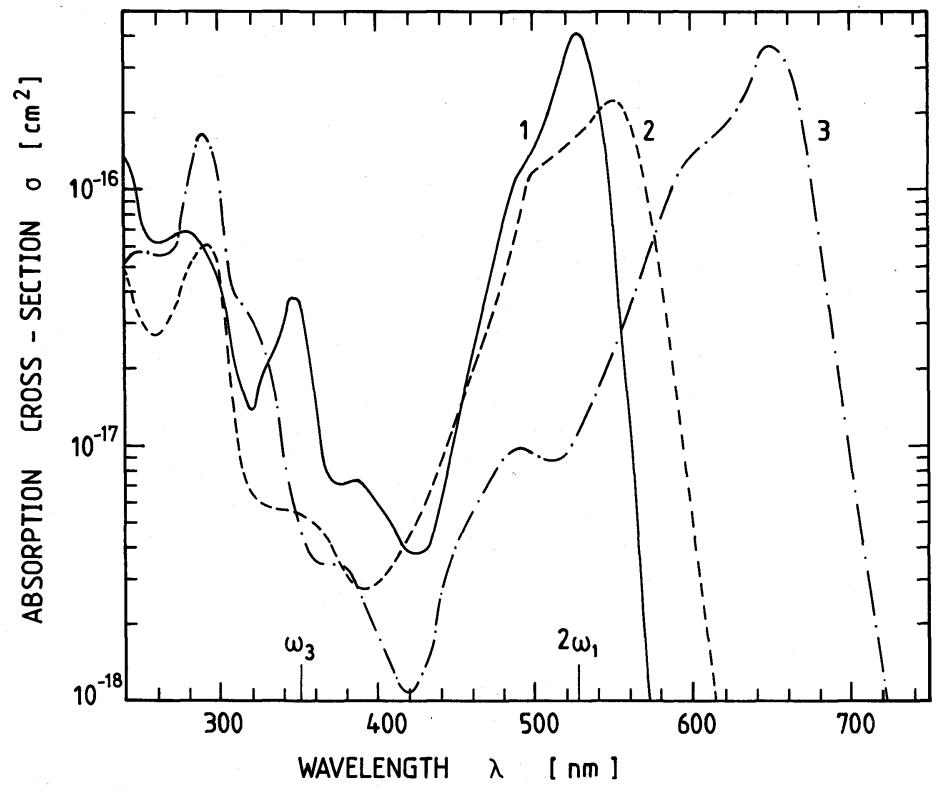

Fig. 1. Absorption spectra of rhodamine 6G (1), fuchsin (2), and methylene blue (3) dissolved in methanol. Concentration $10^{-4} \mathrm{~mol} / \mathrm{dm}^{3}$

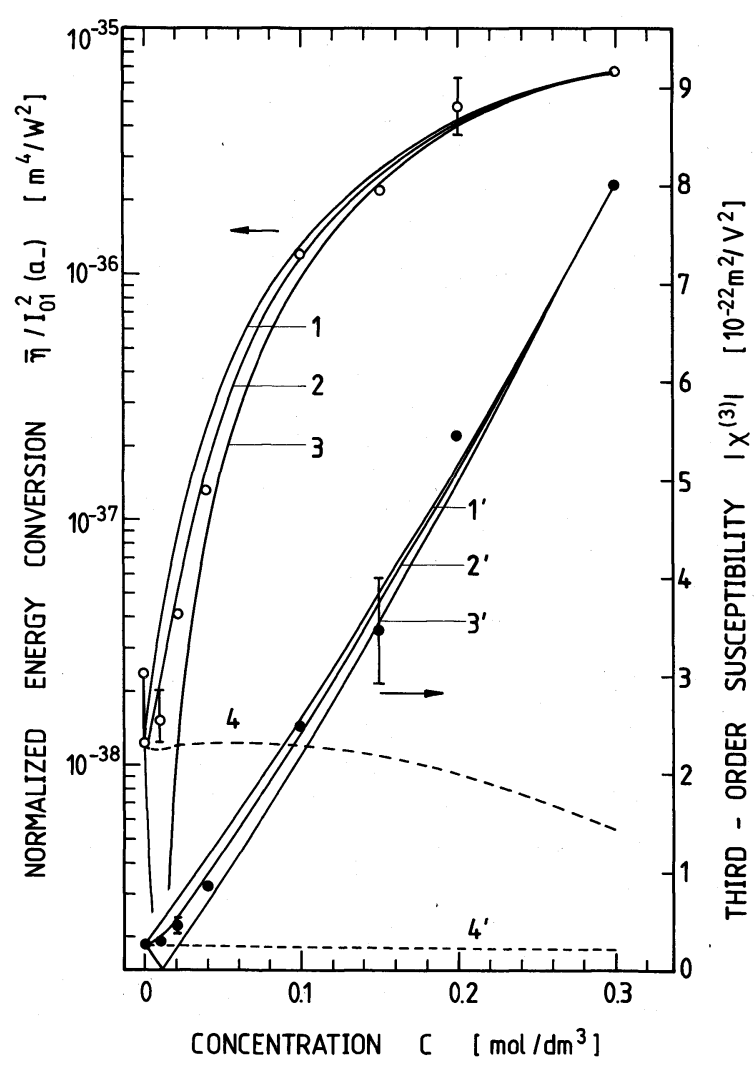

Fig. 2. Energy conversion and third-order nonlinear susceptibility of rhodamine $6 \mathrm{G}$ in methanol versus concentration. Open circles, measured energy conversion. Closed circles, calculated nonlinear susceptibilities from measured energy conversion. Curves are calculated: $1-4$ belong to $\bar{\eta} / I_{01}^{2}\left(a_{-}\right), 1^{\prime}-4^{\prime}$ belong to $\left|\chi^{(3)}\right| .1,1^{\prime}: \gamma_{\mathrm{D}}^{(3)}=1.33 \times 10^{-59} \mathrm{Cm}^{4} / \mathrm{V}^{3}$; $2,2^{\prime}: \gamma_{\mathrm{D}}^{(3)}= \pm \mathrm{i} 1.37 \times 10^{-59} \mathrm{Cm}^{4} / \mathrm{V}^{3} ; 3,3^{\prime}: \gamma_{\mathrm{D}}^{(3)}=-1.41 \times 10^{-59}$ $\mathrm{Cm}^{4} / \mathrm{V}^{3} ; 4,4^{\prime}$ solvent methanol $\gamma_{\mathrm{S}}^{(3)}=\gamma_{\mathrm{SO}}^{(3)}\left(\varrho-C M_{\mathrm{D}}\right) / \varrho_{0}$, $\gamma_{\mathrm{sO}}^{(3)}=6.27 \times 10^{-63} \mathrm{Cm}^{4} / \mathrm{V}^{3}, \quad \varrho_{0}=0.79132 \mathrm{~g} / \mathrm{cm}^{3}, \quad M_{\mathrm{D}}=479.02$ $\mathrm{g} / \mathrm{mol}, \varrho(C=0.2 \mathrm{~mol} / 1)=0.8239 \mathrm{~g} / \mathrm{cm}^{3}$. Sample length $l_{B}=2 \mathrm{~mm}$

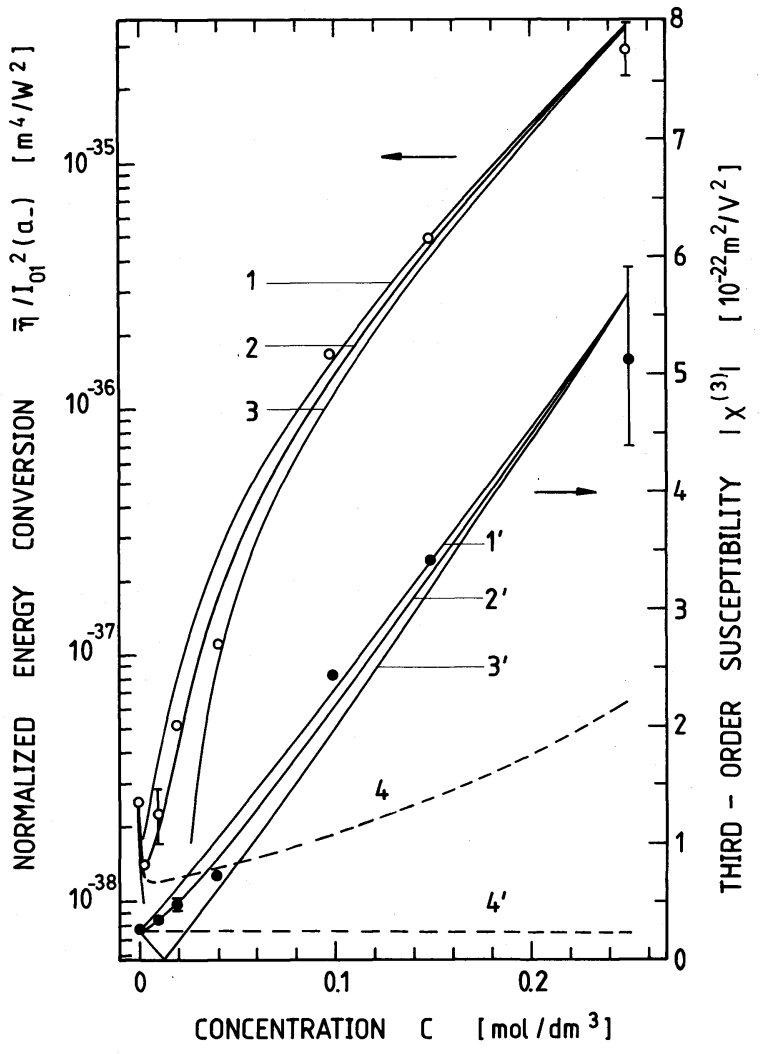

Fig. 3. Energy conversion and third-order nonlinear susceptibility of fuchsin in methanol. Arrangement as in Fig. 2. Curves 1, $1^{\prime}: \gamma_{\mathrm{D}}^{(3)}=1.19 \times 10^{-59} \mathrm{Cm}^{4} / \mathrm{V}^{3} ; 2,2^{\prime}: \gamma_{\mathrm{D}}^{(3)}= \pm \mathrm{i} 1.24$ $\times 10^{-59} \mathrm{Cm}^{4} / \mathrm{V}^{3} ; 3,3^{\prime}: \gamma_{\mathrm{D}}^{(3)}=-1.29 \times 10^{-59} \mathrm{Cm}^{4} / \mathrm{V}^{3} ; 4,4^{\prime}$ methanol, $\quad M_{\mathrm{D}}=337.85 \mathrm{~g} / \mathrm{mol}, \quad \varrho\left(C=0.2 \mathrm{~mol} / \mathrm{dm}^{3}\right)=0.8067 \mathrm{~g} / \mathrm{cm}^{3}$. Sample length $l_{B}=2 \mathrm{~mm}$ 


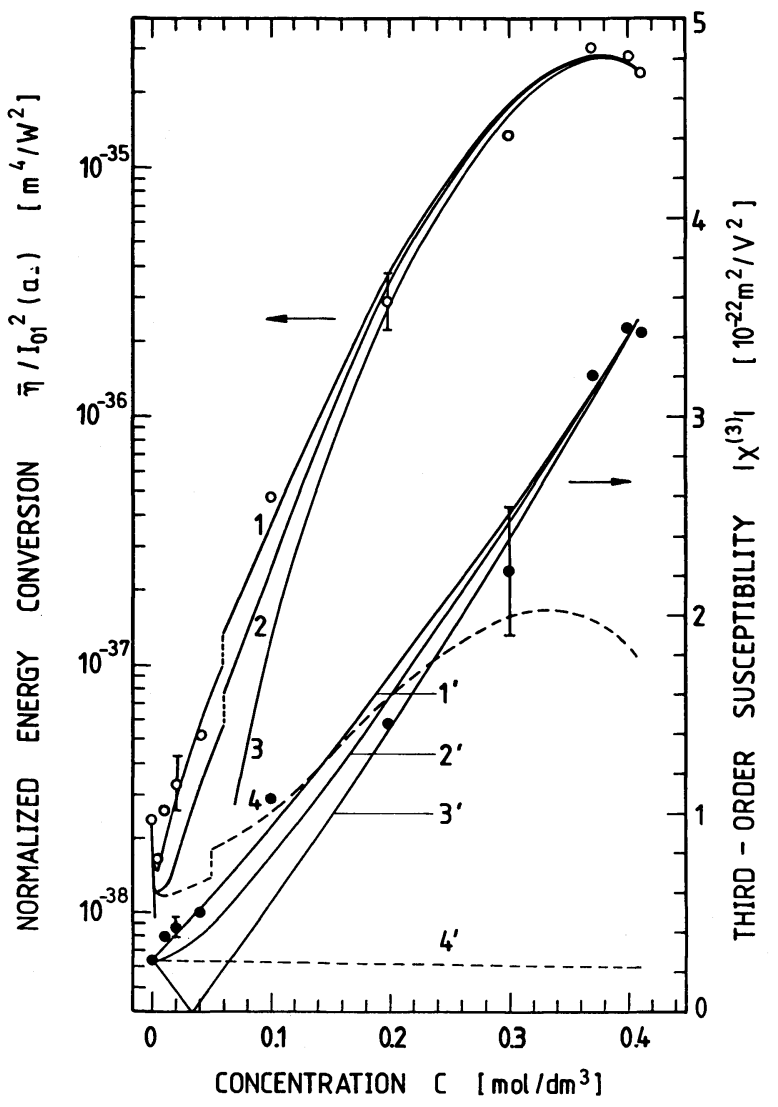

Fig. 4. Energy conversion and third-order nonlinear susceptibility of methylene blue in methanol. Arrangement as in Fig. 2. Curve $1,1^{\prime}: \gamma_{\mathrm{D}}^{(3)}=3.99 \times 10^{-60} \mathrm{Cm}^{4} / \mathrm{V}^{3} ; 2,2^{\prime}: \gamma_{\mathrm{D}}^{(3)}= \pm \mathrm{i} 4.27$ $\times 10^{-60} \mathrm{Cm}^{4} / \mathrm{V}^{3} ; \quad 3,3^{\prime}: \quad \gamma_{\mathrm{D}}^{(3)}=-4.57 \times 10^{-60} \mathrm{Cm}^{4} / \mathrm{V}^{3} ; 4,4^{\prime}$ : methanol, $M_{\mathrm{D}}=355.89, \varrho\left(0.2 \mathrm{~mol} / \mathrm{dm}^{3}\right)=0.8162 \mathrm{~g} / \mathrm{cm}^{3}$. Sample length $l_{B}=2 \mathrm{~mm}$ for $C \leqq 0.06 \mathrm{~mol} / \mathrm{dm}^{3}$, otherwise $l_{B}=0.2 \mathrm{~mm}$

case of methylene blue they rise sublinearly in agreement with [6] (absorption spectrum deviates from Beer's law). The effective interaction length $l_{\text {eff }}=\left[\Delta k^{2}\right.$ $\left.+\left(\alpha_{3}-3 \alpha_{1}\right)^{2} / 4\right]^{-1 / 2}$ is included in Fig. 6 .

The experimental third-order susceptibility values $\left|\chi^{(3)}\right|$ are calculated from the measured $\bar{\eta}$ values with the aid of (2). The obtained values are depicted by closed circles in Figs. 2-4. Their uncertainty $\Delta\left|\chi^{(3)}\right| /\left|\chi^{(3)}\right| \approx 0.15$ follows from the uncertainty of the $\bar{\eta} / I_{01}^{2}\left(a_{-}\right)$values.

The nonlinear susceptibility $\chi^{(3)}$ comprises contributions from the solvent (S) and the solute (D). It may be written as

$\chi^{(3)}=\chi_{\mathrm{S}}^{(3)}+\chi_{\mathrm{D}}^{(3)}$.

The solvent susceptibility is

$\chi_{\mathrm{S}}^{(3)}=\chi_{\mathrm{SO}}^{(3)} \frac{N_{\mathrm{S}}}{N_{\mathrm{SO}}}=\chi_{\mathrm{SO}}^{(3)} \frac{\varrho-N_{\mathrm{D}} M_{\mathrm{D}} / N_{\mathrm{A}}}{\varrho_{0}}$,

where $\chi_{\mathrm{SO}}^{(3)}$ is the nonlinear susceptibility of the neat solvent. It is real and positive (far off resonances). $N_{\mathrm{S}}$ and $N_{\mathrm{D}}=C N_{\mathrm{A}}$ indicate the number densities of solvent and dye molecules, respectively. $N_{\mathrm{A}}=6.022169$ $\times 10^{23} \mathrm{~mol}^{-1}$ is the Avogadro number. $N_{\mathrm{so}}$ is the number density of the neat solvent. $\varrho$ and $\varrho_{0}$ are the mass densities. $M_{\mathrm{D}}$ is the molar mass of the dye molecules. The densities were measured with a pycnometer.

$\chi_{\mathrm{D}}^{(3)}$ is the apparent nonlinear susceptibility of the dye [11]. It includes the intrinsic contribution of the dye molecules and the contribution of the dye-solvent interaction [11]. In general, $\chi_{\mathrm{D}}^{(3)}$ is complex and may be written as

$\chi_{\mathrm{D}}^{(3)}=\chi_{\mathrm{D}}^{(3)^{\prime}}-\mathrm{i} \chi_{\mathrm{D}}^{(3)^{\prime \prime}}=\left|\chi_{\mathrm{D}}^{(3)}\right| \exp (-\mathrm{i} \varphi)$.

It may be expressed in terms of the apparent hyperpolarizability $\gamma_{D}^{(3)}=\left|\gamma_{D}^{(3)}\right| \exp (-i \varphi)$ of the dye by

$$
\begin{aligned}
& \chi_{\mathrm{D}, \boldsymbol{x x} x \boldsymbol{x}}^{(3)}\left(-\omega_{3} ; \omega_{1}, \omega_{1}, \omega_{1}\right) \\
& \quad=\frac{N_{\mathrm{D}} L^{(4)}}{\varepsilon_{0}} \gamma_{\mathrm{D}, x x \boldsymbol{x} x}^{(3)}\left(-\omega_{3} ; \omega_{1}, \omega_{1}, \omega_{1}\right),
\end{aligned}
$$

where $L^{(4)}=\left(n_{3}^{2}+2\right)\left(n_{1}^{2}+2\right)^{3} / 81$ is the Lorentz-localfield correction factor. The definition of $\gamma_{\mathrm{D}}^{(3)}$ follows [12], it differs from the previously used definition in [11] taken from [13] by $\gamma_{D}^{(3)}([12])=\gamma_{D}^{(3)}([11]) / 24$.

The solid curves for $\bar{\eta} / I_{01}^{2}\left(a_{-}\right)$and $\left|\chi^{(3)}\right|$ in Figs. 2-4 are calculated by fitting $\gamma_{D}^{(3)}$ to the experimental energy conversion measurement at the highest dye concentration. The phase angles $\varphi$ are set to $\varphi=0$ (curves 1 and $1^{\prime}, \gamma_{D}^{(3)}$ positive), $\varphi= \pm 90^{\circ}$ (curves 2 and $2^{\prime}, \gamma_{D}^{(3)}$ imaginary, \pm sign undetermined) and $\varphi=180^{\circ}$ (curves 3 and $3^{\prime}, \gamma_{D}^{(3)}$ negative). The concentration dependence of $\bar{\eta} / I_{01}^{2}\left(a_{-}\right)$or of $\left|\chi^{(3)}\right|$ at low concentrations $\left(C \lesssim 0.04 \mathrm{~mol} / \mathrm{dm}^{3}\right)$ allows to determine the phase of the complex hyperpolarizability and susceptibility. The best fitting $\gamma_{\mathrm{D}}^{(3)}$ values are listed in Table 1 . The dashed curves 4 and $4^{\prime}$ in Figs. 2-4 indicate the contributions of the solvent to $\bar{\eta} / I_{01}^{2}\left(a_{-}\right)$and $\left|\chi^{(3)}\right|$. The solvent contribution $\bar{\eta}_{\mathrm{S}} / I_{01}^{2}\left(a_{-}\right)$grows quadratically with the effective interaction length $l_{\text {eff }}$. The dye contribution $\bar{\eta}_{\mathrm{D}}$ at phasematching is independent of the actual dye concentration, since $\bar{\eta}_{\mathrm{D}} \propto\left|\chi_{\mathrm{D}}^{(3)}\right|^{2} / \alpha_{3}^{2}$ with $\left|\chi_{\mathrm{D}}^{(3)}\right| \propto C$ and $\alpha_{3} \propto C$.

\section{Discussion}

The experimental findings listed in Table 1 are:

i) The absolute hyperpolarizability $\left|\gamma_{\mathrm{D}}^{(3)}\right|$ is nearly the same for all three dyes despite the fact that rhodamine 6G has two resonances (absorption peaks near $2 \omega_{1}$ and $\omega_{3}$ ), fuchsin has one resonance (absorption band at $\left.2 \omega_{1}\right)$, while methylene blue has no resonance.

ii) The dye hyperpolarizabilities $\left|\gamma_{\mathrm{D}}^{(3)}\right|$ are about a factor of 1000 larger than the solvent hyperpolarizability $\gamma_{\mathrm{s}}^{(3)}$. 


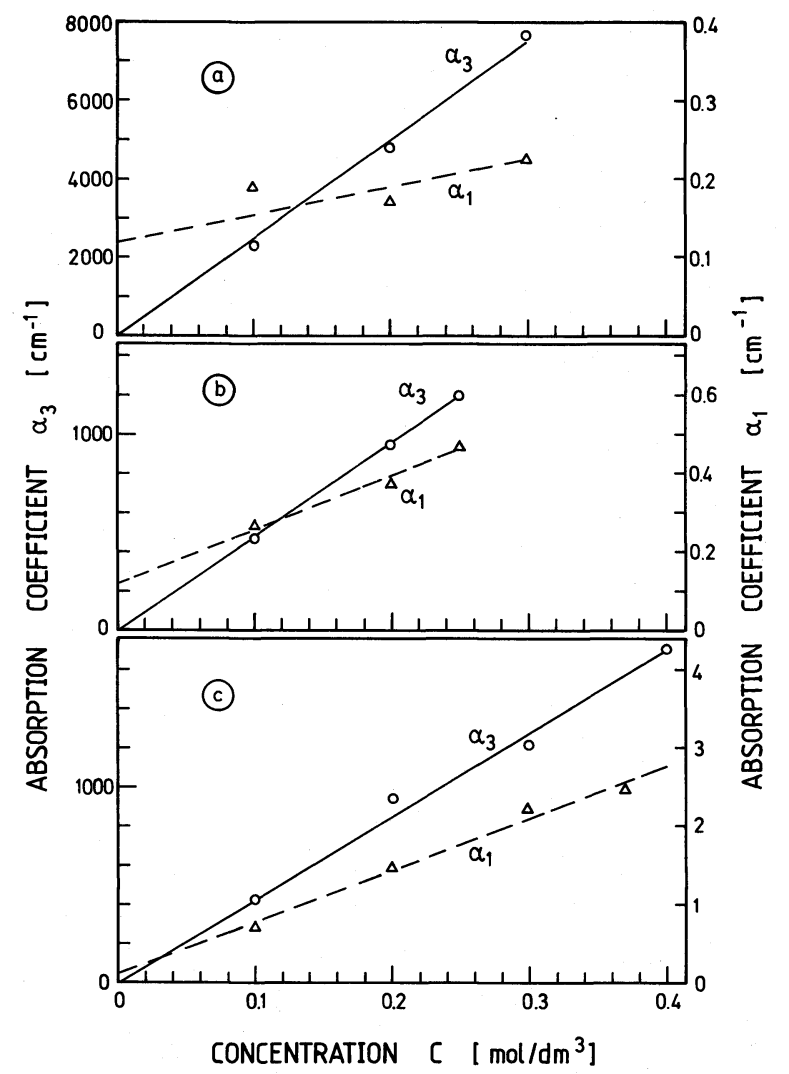

Fig. 5a-c. Absorption coefficients at $\omega_{1}(\Delta)$ and $\omega_{1}(0)$ versus dye concentration. (a) Rhodamine $6 \mathrm{G}$ in methanol. (b) Fuchsin in methanol. (c) Methylene blue in methanol

Table 1

\begin{tabular}{|c|c|c|c|}
\hline \multirow[t]{3}{*}{ Substances } & \multicolumn{3}{|c|}{ Hyperpolarizability } \\
\hline & & & $\varphi$ \\
\hline & {$\left[\mathrm{Cm}^{4} / \mathrm{V}^{3}\right]$} & {$[\mathrm{esu}]^{\mathrm{a})}$} & {$\left[{ }^{\circ}\right]$} \\
\hline Methanol & $6 \times 10^{-63}$ & $5 \times 10^{-38}$ & 0 \\
\hline Rhodamine $6 \mathrm{G}$ & $1.4 \times 10^{-59}$ & $1.1 \times 10^{-34}$ & $110 \pm 30$ \\
\hline Fuchsin & $1.2 \times 10^{-59}$ & $1.0 \times 10^{-34}$ & $90 \pm 30$ \\
\hline Methylene blue & $4 \times 10^{-60}$ & $3.2 \times 10^{-35}$ & $0 \pm 30$ \\
\hline
\end{tabular}

Transformation factors are $\gamma^{(3)}(\mathrm{esu})=8.0888 \times 10^{24} \gamma^{(3)}(\mathrm{SI})$ and $\chi^{(3)}(\mathrm{esu})=\left(9 \times 10^{8} / 4 \pi\right) \chi^{(3)}(\mathrm{SI})[11]$

iii) The hyperpolarizability of rhodamine $6 \mathrm{G}$ and fuchsin is mainly imaginary while the hyperpolarizability of methylene blue is mainly real.

The experimental results are analyzed in the following. The anharmonic oscillator model [11] is inappropriate since it does not cope with the two-photon resonances at $2 \omega_{1}$ : It leads to

$$
\begin{aligned}
& \gamma^{(3)}\left(-\omega_{3} ; \omega_{1}, \omega_{1}, \omega_{1}\right) \\
& \quad=\left(\xi m / 4 f^{2} e^{4}\right) \gamma^{(1)}\left(\omega_{1}\right)^{3} \gamma^{(1)}\left(\omega_{3}\right)
\end{aligned}
$$

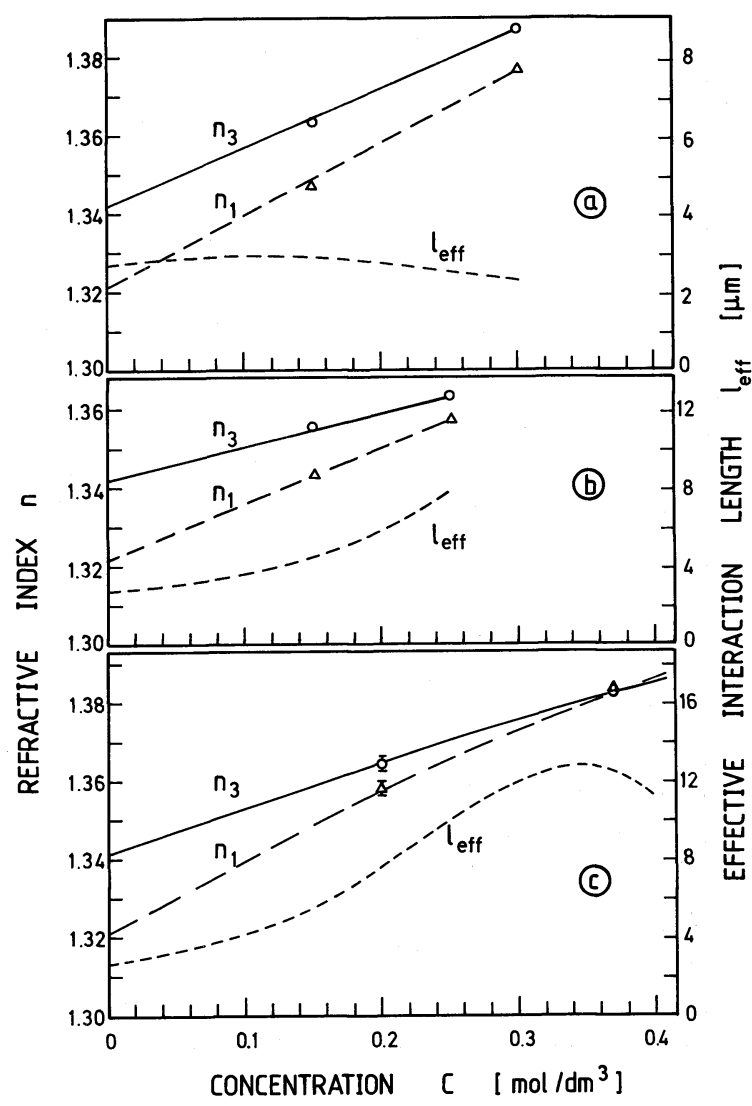

Fig. 6a-c. Refractive indices relative to air at $\omega_{1}(\Delta)$ and $\omega_{3}(0)$ versus dye concentration. $T=25^{\circ} \mathrm{C}, \quad \lambda_{1}=1.055 \mathrm{~nm}$, $\lambda_{3}=351.7 \mathrm{~nm}$. (a) Rhodamine $6 \mathrm{G}$ in methanol. (b) Fuchsin in methanol. (c) Methylene blue in methanol. The effective interaction lengths $l_{\text {eff }}=\left[\left(\alpha_{3}-3 \alpha_{1}\right)^{2} / 4+\Delta k^{2}\right]^{-1 / 2}$ are included

where $\xi$ is the anharmonic coupling constant, $m$ the electron mass, $f$ the oscillator strength, $e$ the electron charge and $\gamma^{(1)}\left(\omega_{i}\right)$ the linear polarizability. $\gamma^{(1)}\left(2 \omega_{1}\right)$ is not involved.

The quantum-mechanical perturbation theory for electric dipole interaction is applied here. A general expression for the hyperpolarizability $\gamma_{x x x x}^{(3)}\left(-\omega_{3} ; \omega_{1}\right.$, $\left.\omega_{1}, \omega_{1}\right)$ is found by third order perturbation theory. The result is [14-17]

$$
\begin{aligned}
\gamma_{x x x x}^{(3)}( & \left.-\omega_{3} ; \omega_{1}, \omega_{1}, \omega_{1}\right) \\
= & \frac{e^{4}}{\hbar^{3}} \sum_{a} \sum_{b} \sum_{c}\left[\frac{\langle g|x| a\rangle\langle a|x| b\rangle\langle b|x| c\rangle\langle c|x| g\rangle}{\left(\omega_{a}-\omega_{3}\right)\left(\omega_{b}-2 \omega_{1}\right)\left(\omega_{c}-\omega_{1}\right)}\right. \\
& +\frac{\langle g|x| a\rangle\langle a|x| b\rangle\langle b|x| c\rangle\langle c|x| g\rangle}{\left(\omega_{a}^{*}+\omega_{1}\right)\left(\omega_{b}-2 \omega_{1}\right)\left(\omega_{c}-\omega_{1}\right)} \\
& +\frac{\langle g|x| c\rangle\langle c|x| b\rangle\langle b|x| a\rangle\langle a|x| g\rangle}{\left(\omega_{a}^{*}+\omega_{3}\right)\left(\omega_{b}^{*}+2 \omega_{1}\right)\left(\omega_{c}^{*}+\omega_{1}\right)} \\
& \left.+\frac{\langle g|x| c\rangle\langle c|x| b\rangle\langle b|x| a\rangle\langle a|x| g\rangle}{\left(\omega_{a}-\omega_{1}\right)\left(\omega_{b}^{*}+2 \omega_{1}\right)\left(\omega_{c}^{*}+\omega_{1}\right)}\right] .
\end{aligned}
$$


The three summations run over all states of the system (ground state $|g\rangle$ and excited states). $\omega_{a}, \omega_{b}$, and $\omega_{c}$ are complex quantities, i.e. $\omega_{\alpha}=\Omega_{\alpha}-\mathrm{i} \Gamma_{\alpha}$ with $\alpha=a, b$, or $c$. The real part $\Omega_{\alpha}$ is equal to a transition frequency from the ground state $|g\rangle$ to an excited state $|\alpha\rangle$, the imaginary part $\Gamma_{\alpha}$ is equal to the half width at half maximum of the absorption line at frequency $\Omega_{\alpha}$ and describes the relaxation processes. Each of the four terms in the bracket contains a product of four matrix elements in the numerator and a product of three frequency expressions in the denominator.

The first of the four sum terms contains three resonance factors $\left(\omega_{a}-\omega_{3}\right),\left(\omega_{b}-2 \omega_{1}\right)$, and $\left(\omega_{c}-\omega_{1}\right)$ while the other three terms have either two, none or one resonance factor. The first term contributes most to $\gamma^{(3)}$ and is only remained. Equation (10) is approximated by

$$
\begin{aligned}
& \gamma_{x x x x}^{(3)}\left(-\omega_{3} ; \omega_{1}, \omega_{1}, \omega_{1}\right) \\
& \approx \frac{e^{4}}{\hbar^{3}} \sum_{a} \sum_{b} \sum_{c} \\
& \cdot \frac{\langle g|x| a\rangle\langle a|x| b\rangle\langle b|x| c\rangle\langle c|x| g\rangle}{\left(\omega_{a}-\omega_{3}\right)\left(\omega_{b}-2 \omega_{1}\right)\left(\omega_{c}-\omega_{1}\right)} .
\end{aligned}
$$

Equation (11) may be further simplified by retaining in the triple sum only those transition frequencies that are nearly resonant to the frequencies $\omega_{3}, 2 \omega_{1}$, or $\omega_{1}$ and therefore contribute most. This procedure leads to the following expressions for the three investigated dyes:

i) Rhodamine $6 \mathrm{G}$

$$
\begin{aligned}
& \gamma_{\mathrm{D}, x x x x}^{(3)}\left(-\omega_{3} ; \omega_{1}, \omega_{1}, \omega_{1}\right) \\
& \approx \frac{e^{4}}{\hbar^{3}}\left(\frac{\langle g|x| 348\rangle\langle 348|x| 530\rangle}{\left(\omega_{348}-\omega_{3}\right)\left(\omega_{530}-2 \omega_{1}\right)}\right. \\
&+\left.\frac{\langle g|x| 280\rangle\langle 280|x| 530\rangle}{\left(\omega_{280}-\omega_{3}\right)\left(\omega_{530}-2 \omega_{1}\right)}\right) \sum_{c} \frac{\langle 530|x| c\rangle\langle c|x| g\rangle}{\omega_{c}-\omega_{1}} \\
& \approx \frac{e^{4}}{\hbar^{3}}\left(-\frac{\langle g|x| 348\rangle\langle 348|x| 530\rangle}{\Gamma_{348} \Gamma_{530}}\right. \\
&\left.+\mathrm{i} \frac{\langle g|x| 280\rangle\langle 280|x| 530\rangle}{\left(\Omega_{280}-\omega_{3}\right) \Gamma_{530}}\right) \\
& \cdot \sum_{c} \frac{\langle 530|x| c\rangle\langle c|x| g\rangle}{\Omega_{c}-\omega_{1}} \\
&= \frac{e^{4}}{\hbar^{3}}\left(-\frac{\langle g|x| 348\rangle\langle 348|x| 530\rangle K_{R}}{\Gamma_{348} \Gamma_{530}\left(\Omega_{348}-\omega_{1}\right)}\right. \\
&\left.+\mathrm{i} \frac{\langle g|x| 280\rangle\langle 280|x| 530\rangle K_{R}}{\left(\Omega_{280}-\omega_{3}\right) \Gamma_{530}\left(\Omega_{348}-\omega_{1}\right)}\right) .
\end{aligned}
$$

The retained transition frequencies are indicated by the corresponding wavelength numbers in $\mathrm{nm}$ (Fig. 1). The enlarged $\left|\gamma_{\mathrm{D}}^{(3)}\right|$-value compared to the solvent is due to the resonance enhancement at $\omega_{3}$ and $2 \omega_{1}$. Since the transition dipole moment $\langle g|x| 348\rangle$ is smaller than $\langle g|x| 280\rangle$ (smaller absorption cross section) the second term in the bracket of $(12 \mathrm{~b})$ dominates and $\gamma_{\mathrm{D}}^{(3)}$ is complex with a greater imaginary than negative real part. This behaviour is found experimentally. In (12c) the lowest non-vanishing frequency term $\left(\Omega_{348}-\omega_{1}\right)$ is singled out and the remaining quantity is abreviated by the constant

$K_{R}=\sum_{c}\langle 530|x| c\rangle\langle c|x| g\rangle\left(\Omega_{348}-\omega_{1}\right) /\left(\Omega_{c}-\omega_{1}\right)$.

ii) Fuchsin

$$
\begin{aligned}
\gamma_{\mathrm{D}, x x x x}^{(3)} & \left(-\omega_{3} ; \omega_{1}, \omega_{1}, \omega_{1}\right) \\
\approx & \frac{e^{4}}{\hbar^{3}} \frac{\langle g|x| 290\rangle\langle 290|x| 550\rangle}{\left(\omega_{290}-\omega_{3}\right)\left(\omega_{550}-2 \omega_{1}\right)} \\
& \cdot \sum_{c} \frac{\langle 550|x| c\rangle\langle c|x| g\rangle}{\omega_{c}-\omega_{1}} \\
\approx & \mathrm{i} \frac{e^{4}}{\hbar^{3}} \frac{\langle g|x| 290\rangle\langle 290|x| 550\rangle}{\left(\Omega_{290}-\omega_{3}\right) \Gamma_{550}} \\
& \cdot \sum_{c} \frac{\langle 550|x| c\rangle\langle c|x| g\rangle}{\Omega_{c}-\omega_{1}} \\
= & \mathrm{i} \frac{e^{4}}{\hbar^{3}} \frac{\langle g|x| 290\rangle\langle 290|x| 550\rangle K_{F}}{\left(\Omega_{290}-\omega_{3}\right) \Gamma_{550}\left(\Omega_{290}-\omega_{1}\right)} .
\end{aligned}
$$

$K_{F}$ is given by

$K_{F}=\sum_{c}\langle 550|x| c\rangle\langle c|x| g\rangle\left(\Omega_{290}-\omega_{1}\right) /\left(\Omega_{c}-\omega_{1}\right)$.

The resonant term at $\omega_{a}=350 \mathrm{~nm}$ is neglected since the transition dipole moment $\langle g|x| 350\rangle$ is very small (very small absorption at $350 \mathrm{~nm}$, compared to $290 \mathrm{~nm}$, see Fig. 1). The $\left|\gamma_{\mathrm{D}}^{(3)}\right|$ value is enhanced by the two-photon resonance at $2 \omega_{1}$ and the small difference frequency term $\Omega_{290}-\omega_{3}\left[\left(\Omega_{290}-\omega_{3}\right) / \Gamma_{550} \approx 3\right]$. Equation (13) is imaginary in agreement with the experimental findings (Table 1).

iii) Methylene blue

$$
\begin{aligned}
& \gamma_{\mathrm{D}, \boldsymbol{x} x \boldsymbol{x}}^{(3)}\left(-\omega_{3} ; \omega_{1}, \omega_{1}, \omega_{1}\right) \\
& \approx \frac{e^{4}}{\hbar^{3}} \frac{\langle g|x| 290\rangle}{\omega_{290}-\omega_{3}}\left(\sum_{b} \frac{\langle 290|x| b\rangle\langle b|x| 650\rangle}{\omega_{b}-2 \omega_{1}}\right) \\
& \cdot \frac{\langle 650|x| g\rangle}{\omega_{650}-\omega_{1}} \\
& \approx \frac{e^{4}}{\hbar^{3}} \frac{\langle g|x| 290\rangle\langle 650|x| g\rangle}{\left(\Omega_{290}-\omega_{3}\right)\left(\Omega_{650}-\omega_{1}\right)} \\
& \cdot \sum_{b} \frac{\langle 290|x| b\rangle\langle b|x| 650\rangle}{\Omega_{b}-2 \omega_{1}} \\
&= \frac{e^{4}}{\hbar^{3}} \frac{\langle g|x| 290\rangle\langle 650|x| g\rangle K_{M}}{\left(\Omega_{290}-\omega_{3}\right)\left(\Omega_{650}-\omega_{1}\right) 2 \omega_{1}} .
\end{aligned}
$$


$K_{M}$ represents the constant

$K_{m}=\sum_{b}\langle 290|x| b\rangle\langle b|x| 650\rangle 2 \omega_{1} /\left(\Omega_{b}-2 \omega_{1}\right)$.

$\gamma_{\mathrm{D}}^{(3)}$ is positive. For a crude comparison of the hyperpolarizability of methylene blue with fuchsin one may compare the resonance denominators leading to $\gamma_{D}^{(3)}$

(methylene blue) $/ \mid \gamma_{\mathrm{D}}^{(3)}$ (fuchsin) $\mid$

$$
\approx \Gamma_{550}\left(\Omega_{290}-\omega_{1}\right) /\left[\left(\Omega_{650}-\omega_{1}\right) 2 \omega_{1}\right] \approx 0.29 \text {. }
$$

This ratio is in good agreement with the experimental ratio $\gamma_{\mathrm{D}}^{(3)}$ (methylene blue) $/ \mid \gamma_{\mathrm{D}}^{(3)}$ (fuchsin) $\mid \approx 0.33$. Due to the broad absorption bands of the dyes, $\gamma_{\mathrm{D}}^{(3)}$ remains large even if frequencies $\omega_{1}, 2 \omega_{1}$, and $\omega_{3}$ do not coincide with transition frequency $\Omega_{\alpha}$.

An order of magnitude estimate of the ratio $\left|\gamma_{\mathrm{D}}^{(3)}\right| / \gamma_{\mathrm{S}}^{(3)}$ for the investigated dyes and the solvent methanol may be found by comparing the frequency denominators

$\frac{\left|\gamma_{\mathrm{D}}^{(3)}\right|}{\gamma_{\mathrm{S}}^{(3)}} \approx \frac{\left(\Omega_{\mathrm{S}}-3 \omega_{1}\right)\left(\Omega_{\mathrm{S}}-2 \omega_{1}\right)\left(\Omega_{\mathrm{S}}-\omega_{1}\right)}{\left|\left(\omega_{\mathrm{D} 3}-\omega_{3}\right)\left(\omega_{\mathrm{D} 2}-2 \omega_{1}\right)\left(\omega_{\mathrm{D} 1}-\omega_{1}\right)\right|}$.

$\Omega_{\mathrm{S}}$ is an effective transition frequency of the solvent lying near to the lowest strong electronic absorption band. $\omega_{\mathrm{Di}}=\Omega_{\mathrm{Di}}-\mathrm{i} \Gamma_{\mathrm{Di}}$ represent the relevant dye transition frequencies of (12c) to (14c). Setting two frequency factors of the dyes equal to $1000 \mathrm{~cm}^{-1}$ and the third one equal to $10000 \mathrm{~cm}^{-1}$ and using the experimental ratio $\left|\gamma_{\mathrm{D}}^{(3)}\right| / \gamma_{\mathrm{S}}^{(3)} \approx 1000$, one obtains an effective solvent transition frequency of $\tilde{v}_{\mathrm{S}}=\Omega_{\mathrm{S}} /\left(2 \pi c_{0}\right) \approx 46000 \mathrm{~cm}^{-1}\left(\lambda_{\mathrm{S}}=217 \mathrm{~nm}\right)$. Near this frequency methanol has its first electronic absorption band. Equation (15) gives a reasonable result.

An enlargement of $\gamma_{\mathrm{D}}^{(3)}$ due to conjugated double bonds was discussed in [18-21]. Here we need the conjugated double bonds only to bring the $S_{1}$ absorption band to the visible region between $\omega_{1}$ and $\omega_{3}$. Enlargement of $\gamma_{\mathrm{D}}^{(3)}$ beyond that expected from the resonance denominators is not observed.

\section{Conclusions}

The third-order nonlinear susceptibility of dye solutions versus concentration was analysed. A special arrangement was used to avoid disturbing thirdharmonic generation from the environment. At low dye concentrations the energy conversion is sensitive to amplitude and phase of the complex dye hyper- polarizability. The real and imaginary part of the hyperpolarizability were determined. Theoretical expressions for the hyperpolarizability derived from third-order perturbation theory explain the experimental findings. The enlarged dye hyperpolarizability compared to the solvent $\left(\left|\gamma_{\mathrm{D}}^{(3)}\right| / \gamma_{\mathrm{S}}^{(3)} \approx 1000\right)$ is well understood by the broad absorption bands at or near to the involved frequencies $\omega_{1}, 2 \omega_{1}$, and $\omega_{3}=3 \omega_{1}$.

Acknowledgements. The authors are grateful to $\mathrm{Mr}$. $\mathrm{H}$. J. Lehmeier for his help in the final experiments and to Mr. Th. Ascherl for technical assistance. They thank the "Deutsche Forschungsgemeinschaft" for financial support and the "Rechenzentrum" of the Regensburg University for disposal of computer time.

\section{References}

1. P.P. Bey, I.E. Giuliani, H. Rabin: Phys. Rev. Lett. 19, 819 (1967)

2. P.P. Bey, I.E. Giuliani, H. Rabin: IEEE J. QE-4, 932 (1968)

3. R.K. Chang, L.K. Galbraith: Phys. Rev. 171, 993 (1968)

4. P.P. Bey, J.F. Giuliani, H. Rabin: IEEE J. QE-7, 86 (1971)

5. J.C. Diels, F.P. Schäfer: Appl. Phys. 5, 197 (1974)

6. L.I. Al'perovich, T.B. Bavaev, V.V. Shabalov: Sov. J. Appl. Spectrosc. 26, 196 (1977)

7. M. Thalhammer, A. Penzkofer: Appl. Phys. B32, 137 (1983)

8. K. Schmid. A. Penzkofer: Appl. Opt. 22, 1824 (1983)

9. W. Leupacher, A. Penzkofer: Appl. Opt. 23, 1554 (1984)

10. D.E. Gray (ed.): American Institute of Physics Handbook, 3rd ed. (McGraw-Hill, New York 1972)

11. A. Penzkofer, J. Schmailzl, H. Glas: Appl. Phys. B29, 37 (1982)

12. R.W. Minck, R.W. Terhune, C.C. Wang: Appl. Opt. 5, 1595 (1966)

13. N.L. Boiling, A.J. Glass, A. Owyoung: IEEE J. QE-14, 601 (1978)

14. P.N. Butcher: Nonlinear Optical Phenomena (Bulletin 200, Engineering Experiment Station, Ohio State University, Columbus, Ohio 1965)

15. R.W. Terhune, P.D. Maker: in Lasers, Vol. 2, ed. by A.K. Levine (Dekker, New York 1968) Chap. 4

16. M. Schubert, B. Wilhelmi: Einführung in die nichtlineare Optik. Teil II. Quantenphysikalische Beschreibung (Teubner, Leipzig 1978)

17. A. Penzkofer, W. Kaiser: Opt. Quant. Electron.9,315(1977)

18. J.P. Hermann, D. Ricard, J. Ducuing: Appl. Phys. Lett. 23, 178 (1973)

19. K.C. Rustagi, J. Ducuing: Opt. Commun. 10, 258 (1974)

20. J.P. Hermann, J. Ducuing: J. Appl. Phys. 45, 5100 (1974)

21. C. Sauteret, J.P. Hermann, R. Frey, F. Pradere, J. Ducuing, R.H. Baughman, R.R. Chance: Phys. Rev. Lett. 36, 956 (1976) 